\title{
The Association of Septic Thrombophlebitis with Septic Pulmonary Embolism in Adults
}

\author{
Jorge A. Brenes ${ }^{*}, 1$, Umesh Goswami ${ }^{1}$ and David N. Williams ${ }^{2}$ \\ ${ }^{I}$ Department of Medicine and ${ }^{2}$ Division of Infectious Diseases, Hennepin County Medical Center, Minneapolis, \\ Minnesota, MN 55415, USA
}

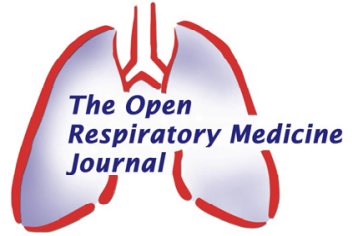

\begin{abstract}
Background: There have been an increasing number of pediatric reports of septic pulmonary embolism in the setting of septic thrombophlebitis adjacent to a primary infectious source.

Methods: Retrospective review at an urban hospital. A total of five adults with a documented primary infectious source, adjacent septic thrombophlebitis and septic pulmonary embolism were identified between 2000 and 2011.

Results: The predominant symptoms on presentation were fever and pleuritic chest pain, followed by chills and cough. $S$. aureus was the pathogen in 4 patients. Only one case had echocardiographic evidence of endocarditis. All patients received IV antibiotics and anticoagulation therapy. No new embolic events or central nervous system complications were noted.

Conclusions: The triad of extrapulmonary infection, contiguous septic thrombophlebitis and septic pulmonary embolism is present in adult as well as pediatric populations. The use of systemic anticoagulation with appropriate antibiotics resulted in clinical and radiologic improvement but no significant complications.
\end{abstract}

Keywords: Septic thrombophlebitis, septic pulmonary emboli, anticoagulation.

\section{INTRODUCTION}

Septic pulmonary embolism (SPE) is a relatively uncommon syndrome characterized by embolization of thrombi containing pathogens admixed with fibrin from an infected site into the venous circulation leading to implantation in the vascular system of the lungs, with secondary infection. In addition to established clinical presentations such as septic thrombophlebitis of the internal jugular vein, septic pelvic thrombophlebitis and right-sided infective endocarditis [1], the triad of an active extrapulmonary source of infection, an adjacent venous thrombosis and septic pulmonary embolism has been recently described in the pediatric literature [2], typically in the setting of deep soft tissue infection and osteomyelitis of the extremities. In order to better understand the dynamics of the disease in adults, we conducted a retrospective analysis of all patients presenting with septic pulmonary embolism to our institution, focusing on those with concomitant septic thrombophlebitis

\section{MATERIALS, METHODS AND RESULTS}

A computer-assisted search of the chest CT imaging database from December 1, 2000 to August 30th, 2011 was performed at Hennepin County Medical Center, a 360-bed urban hospital in Minneapolis, MN. Among all cases identified on chest imaging as having SPE, those patients who had an active primary source of infection and a documented contiguous septic thrombophlebitis were

*Address correspondence to this author at the Hennepin County Medical Center, 701 Park Avenue. Minneapolis, MN 55415, USA; Tel: 612-2054158; Fax: 612-904-4226; E-mail: jorgebrenes80@hotmail.com included in the current study. Septic thrombophlebitis was defined by the following criteria: 1) presence of acute venous thrombosis, as evidenced by ultrasound and/or CT scan and 2) documented bacteremia. The case definition of septic pulmonary embolism required all of the following criteria: 1) suggestive clinical presentation (eg. fever, chills, pleuritic chest pain, dyspnea, hemoptysis, and/or cough) 2) Chest CT with multifocal peripheral nodules suggestive of embolism 3) an extrapulmonary infectious source for emboli, and 4) exclusion of other explanations for lung infiltrates.

Five patients with venous thrombosis contiguous to the infectious focus were identified and characterized in the current study (Table 1).

\section{CASE SERIES}

\section{Case 1}

A 64-year old female with Type 2 diabetes mellitus was admitted with 3 days of progressive confusion, increased urinary frequency and weakness. She had not been taking her medications. She was found to be dehydrated, with laboratory evidence of diabetic ketoacidosis. Aggressive fluid resuscitation and intravenous insulin infusion were started. Chest $\mathrm{X}$-ray was notable for ill defined left lower lobe opacity. Blood cultures grew methicillin-sensitive $S$. aureus (MSSA), and intravenous antibiotic therapy was tailored accordingly. Despite appropriate antimicrobial therapy, blood cultures continued to be positive for 6 days. A transesophageal echocardiogram (TEE) did not reveal evidence of endocarditis. A CT scan of the chest was significant for multiple bilateral pulmonary nodules with peripheral distribution, suggestive of embolic nature. CT of the abdomen and pelvis (Fig. 1B) revealed right sided 
Table 1. Baseline Characteristics, Microbiology, Imaging and Outcomes of Patients with Septic Thrombophlebitis Complicated by Septic Pulmonary Embolism

\begin{tabular}{|c|c|c|c|c|c|}
\hline & Case 1 & Case 2 & Case 3 & Case 4 & Case 5 \\
\hline Age (in years) & 64 & 57 & 31 & 37 & 43 \\
\hline Gender & $\mathrm{F}$ & M & M & $\mathrm{F}$ & M \\
\hline Comorbidities & Type 2 DM & Type 2 DM & Cocaine abuse & Heroin dependence & Heroin dependence \\
\hline $\begin{array}{l}\text { Presenting } \\
\text { symptoms }\end{array}$ & $\begin{array}{l}\text { Confusion, Urinary } \\
\text { frequency }\end{array}$ & $\begin{array}{l}\text { Fever, chills, pleuritic } \\
\text { chest pain }\end{array}$ & $\begin{array}{l}\text { Fever, pleuritic chest } \\
\text { pain }\end{array}$ & $\begin{array}{l}\text { Fever, cough, pleuritic } \\
\text { chest pain }\end{array}$ & $\begin{array}{l}\text { Fever, leg pain, } \\
\text { pleuritic chest pain }\end{array}$ \\
\hline $\begin{array}{l}\text { Extrapulmonary } \\
\text { source }\end{array}$ & Left pyelonephritis & $\begin{array}{l}\text { Vertebral osteomyelitis, } \\
\text { paraspinal abscesses }\end{array}$ & Left thigh pyomyositis & $\begin{array}{l}\text { Right thigh } \\
\text { cellulitis/abscess }\end{array}$ & Left thigh abscess \\
\hline $\begin{array}{c}\text { Pathogen } \\
\text { (isolation sites) }\end{array}$ & MSSA (blood, urine) & MSSA (blood, abscess) & $\begin{array}{c}\text { Group C B-hemolytic } \\
\text { Streptococcus (blood, } \\
\text { bronchoalveolar lavage) }\end{array}$ & $\begin{array}{l}\text { MRSA (blood, } \\
\text { abscess) }\end{array}$ & MSSA (blood, abscess) \\
\hline Thrombosis & $\begin{array}{c}\text { Right renal } \\
\text { vein/inferior Vena cava }\end{array}$ & Azygous vein & Left femoral vein & Right femoral vein & Left femoral vein \\
\hline CXR findings & $\begin{array}{l}\text { Left lower lobe } \\
\text { Consolidation }\end{array}$ & $\begin{array}{l}\text { Right upper lobe } \\
\text { cavitary lesion }\end{array}$ & $\begin{array}{l}\text { Bilateral non specific } \\
\text { infiltrates }\end{array}$ & $\begin{array}{l}\text { Bilateral non specific } \\
\text { infiltrates }\end{array}$ & $\begin{array}{l}\text { Left lower lobe } \\
\text { consolidation }\end{array}$ \\
\hline CT findings & $\begin{array}{c}\text { Bilateral nodules, } \\
\text { effusion, consolidation } \\
\text { left }\end{array}$ & $\begin{array}{l}\text { Bilateral cavitary } \\
\text { nodules, consolidation }\end{array}$ & $\begin{array}{l}\text { Bilateral cavitary } \\
\text { nodules, pleural } \\
\text { effusions }\end{array}$ & $\begin{array}{l}\text { Bilateral cavitary } \\
\text { nodules }\end{array}$ & $\begin{array}{l}\text { Bilateral cavitary } \\
\text { nodules }\end{array}$ \\
\hline Echocardiogram & No vegetations* & No vegetations* & No vegetations & Tricuspid vegetations & No vegetations* \\
\hline $\begin{array}{l}\text { TIA/Stroke, } \\
\text { systemic embolic } \\
\text { phenomena }\end{array}$ & None & None & None & None & None \\
\hline $\begin{array}{l}\text { Hemorrhaghic } \\
\text { complications }\end{array}$ & None & None & Hemopericardium & None & None \\
\hline $\begin{array}{l}\text { Follow up } \\
\text { Imaging }\end{array}$ & Resolving & Resolving & Resolving & Resolving & Resolving \\
\hline
\end{tabular}

pyelonephritis with mild hydronephosis, complicated by a right renal vein thrombosis extending into the inferior vena cava. Anticoagulation with intravenous heparin was started. Systemic inflammatory response gradually defervesced, with resolution of bacteremia. She completed a 6 week course of intravenous antibiotics and 3 months of oral anticoagulation.

\section{Case 2}

A 57-year old male with a new diagnosis of Type 2 diabetes mellitus was admitted from jail with 1 week of right sided pleuritic chest pain, fever and difficulty breathing. He had been involved in a motor vehicle accident two weeks prior to presentation, where he sustained mild contusions to his back. Physical examination revealed an ill appearing man with a fluctuant mass on his left anterior chest and tenderness to palpation over his throracic spine. CXR showed a cavitary nodule in his right upper lobe. Blood cultures were persistently positive for MSSA. CT of the chest showed a chest wall abscess, multiple thick walled, peripheral cavitary lesions, and a paraspinal fluid collection along with extensive thrombosis of the azygous vein (Fig. 1A). Intravenous antibiotics, as well as systemic anticoagulation with heparin were started. MRI of the spine

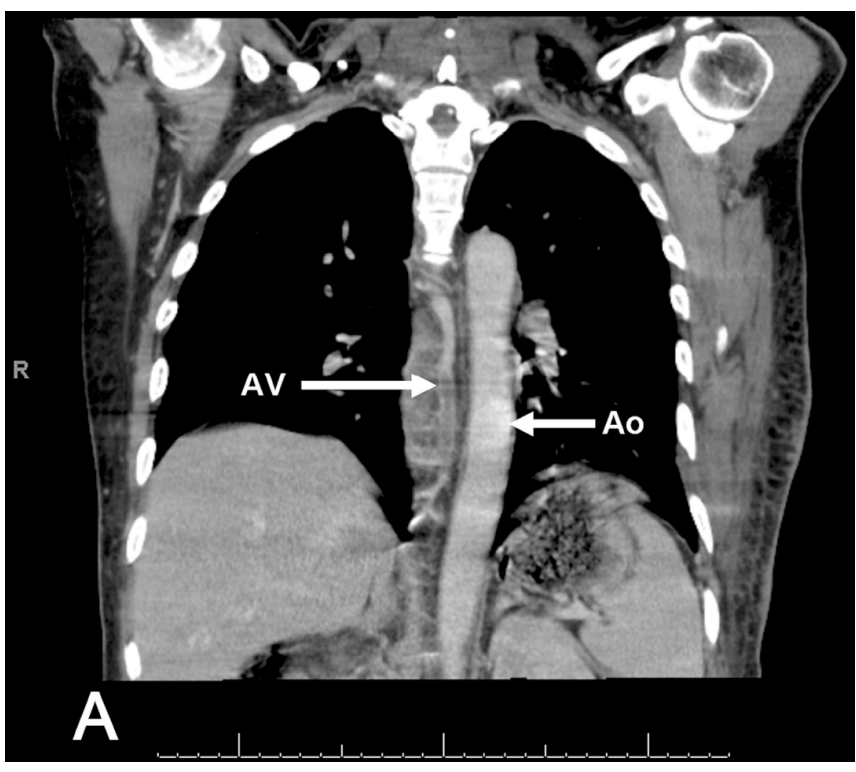

Fig. (1A). CT scan of the chest with IV contrast that revealed the presence of extensive septic thrombophlebitis of the azygos vein $(\mathrm{AV}), \mathrm{Ao}=$ aorta. 


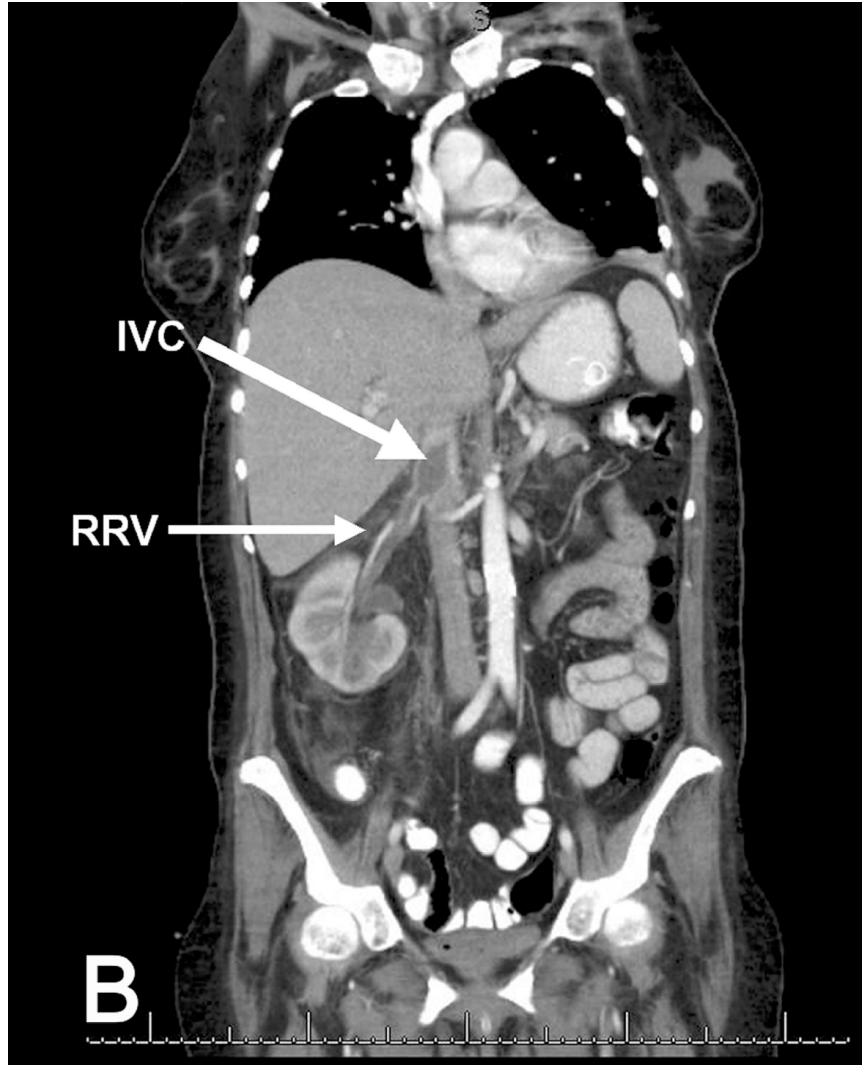

Fig. (1B). Coronal view of CT scan of the abdomen and pelvis with IV contrast that shows acute inflammatory changes in the right kidney consistent with pyelonephritis, complicated by septic thrombophlebitis of the right renal vein (RRV), extending into the inferior vena cava (IVC).

confirmed the presence of a T6-T8 osteomyelitis with multiple small paraspinal abscesses. The patient's clinical condition gradually improved with medical therapy. However, bacteremia persisted until the chest wall abscess was surgically evacuated. He completed a 6 week course of intravenous antibiotics and a 6 month course of oral anticoagulation.

\section{Case 3}

A 31-year old male was brought in by ambulance with complaints of fever and pleuritic chest pain for an unclear period of time. On arrival to the Emergency Department, he was hypothermic and confused, with signs of shock. Primary and secondary surveys were unrevealing. He was intubated due to progressive hypoxemic respiratory failure. CXR showed bilateral non specific pulmonary infiltrates. Septic shock was suspected and broad spectrum antibiotics were started. Blood and bronchoalveolar lavage cultures grew Group C beta- hemolytic Streptococcus. CT images of the chest indicated multiple bilateral cavitary lesions consistent with septic emboli. The patient continued to have persisting fevers with significant oxygen requirements for two weeks. A nuclear medicine gallium scan was obtained and showed marked tracer uptake in several areas, but was particularly intense at the left thigh and right hip. Doppler US of the lower extremities revealed a left common femoral thrombosis, thus anticoagulation with intravenous heparin was started. CT scan revealed an abscess at the border of the left vastus intermedius and vastus lateralis muscles, as well as a larger right hip abscess, which were drained. Three days after initiation of heparin infusion, his cardiac output decreased markedly and he was found to have a hemopericardium; a pericardial window was performed and he remained stable. An inferior vena cava filter was placed; anticoagulation was resumed one week afterwards without further complications. He was able to be weaned from vasopressors and eventually extubated. He made a gradual recovery given initial multiorgan failure, and completed 6 weeks of intravenous antibiotic therapy, as well as a 6 month course of oral anticoagulation.

\section{Case 4}

A 37-year old female with a history of heroin dependence presented to the Emergency Department with 1 week of fever and right thigh pain, along with cough and pleuritic chest pain that began 3 days prior to admission. Physical examination was remarkable for bibasilar crackles, as well as cellulitis of the right proximal thigh, at the level of the femoral vein. Doppler US of the lower extremities revealed thrombosis of the right greater saphenous vein, extending into the right common femoral vein. CXR showed multiple bilateral nodular opacities, which were further characterized by CT scan of the chest as septic emboli. Given the strong suspicion of right sided endocarditis, a transthoracic echocardiogram was performed, but no vegetations were present. Blood cultures were positive for MRSA, thus intravenous antibiotics as well as systemic anticoagulation with heparin were started. The patient appeared to be improving clinically, but she had persistent bacteremia five days after starting appropriate antibiotic therapy. Surgery service was consulted, who noted that an abscess had developed at the initial site of cellulitis. After incision and drainage of the lesion, blood cultures became negative. She completed a 6 week course of intravenous antibiotics and 6 months of oral anticoagulation.

\section{Case 5}

A 43-year old male with a history of heroin dependence was admitted with 3 days of subjective fevers, left thigh pain and pleuritic chest pain. Physical examination revealed an abscess at the level of the left thigh, with multiple injection marks. Doppler US of the lower extremities showed extensive thrombosis of the left greater saphenous, left superficial, left common and left popliteal veins, and confirmed the presence of an abscess anterior to the femoral vessels. CXR showed a non specific left lung base opacity. CT scan of the chest demonstated multiple, peripheral nodules, with a dominant cavitary lesion in the left lower lobe, consistent with septic emboli. Transesophageal echocardiogram was negative for vegetations. Multiple blood cultures were positive for MSSA. He underwent incision and drainage of the left thigh abscess, as well as medical treatment with intravenous antibiotics and heparin. Within 72 hours, his blood cultures became negative, and his condition gradually improved. He completed a 4 week course of intravenous antibiotics and 6 months of oral anticoagulation.

\section{DISCUSSION}

Septic thrombophlebitis (STP) can theoretically affect any vein in the body, both superficial and deep. The 
diagnosis is made based on clinical manifestations, culture data and radiographic evidence of thrombosis. A number of distinct clinical conditions have been identified, depending on the vessel involved, but pathogenic mechanisms tend to be similar. Despite the increased reported incidence of STP related to invasive medical devices or procedures [3], this phenomenon was not observed in the series presented. However, in two patients, use of non sterile needles for intravenous drug use was clearly a factor. A particular high index of suspicion of thrombosis is required in such individuals, where the manifestations of the soft tissue infection (erythema, pain, and swelling) can easily overlap those of STP.

As documented by this retrospective case series, the concurrence of an extrapulmonary source of infection with contiguous septic thrombophlebitis, complicated by septic pulmonary embolism seems to affect the adult population as well. This syndrome has been well established in prior descriptions in children [4-7], particularly with acute osteomyelitis as the primary focus.

In most of our cases, the keys for diagnosis were the presence of septic pulmonary emboli on chest $\mathrm{CT}$ in the setting of persistent bacteremia.

Despite differences in the primary source of infection and location of STP, we postulate that a common pathophysiologic sequence could explain the events in this group of patients (Fig. 2). A local infectious process can cause extravasation or translocation of an organism, typically bacterial, into the venous system. Depending on the extent of the infection, edema could result in venous compression and favor stasis [4]. Once in the bloodstream, damage to the endothelium by toxins and inflammatory mediators, as well as direct production of thrombogenic toxins by the organism eventually leads to thrombosis. The fibrin and platelet matrix serves as an ideal nidus for proliferation of the organism, with potential for propagation, and allows for distant metastatic infection as its contents are drained into the pulmonary circulation.

The culprit organism seems to play a pivotal role in this syndrome. Many of the pathogenetic mechanisms of septic thrombophlebitis have been learned from Fusobacterium necrophorum, the most common causative agent of Lemierre's syndrome. Massive inflammation and altered coagulation are features associated with this organism $[8,9]$. Like $F$. necrophorum, it is known that $S$. aureus has a tendency to promote venous thrombosis. Both bacteria produce an extracellular, heat stable leucocidin (PantonValentine leucocidin for S. aureus) that exhibits thrombogenic effects, through indirect inflammatory mechanisms, including the formation of reactive oxygen species and release of secondary inflammatory mediators from dying granulocytes, causing intense endothelial dysfunction [10]. $S$. aureus produces coagulase, which specifically interacts with fibrinogen and causes coagulation [2]. It is no surprise that several "variants" of Lemierre's syndrome caused by $S$. aureus have been reported [11-13]. We believe that the predominance of $S$. aureus as the causative agent in our series is related to its intrinsic thrombogenic and proinflammatory potential, rather than just its expected relation to the type of primary focus. This is consistent with prior data $[6,7,14,15]$.

The commonly accepted principles of treatment for septic pulmonary embolism in the setting of septic thrombophlebitis include prompt empiric administration of intravenous antibiotics, detecting and removing any potentially infected

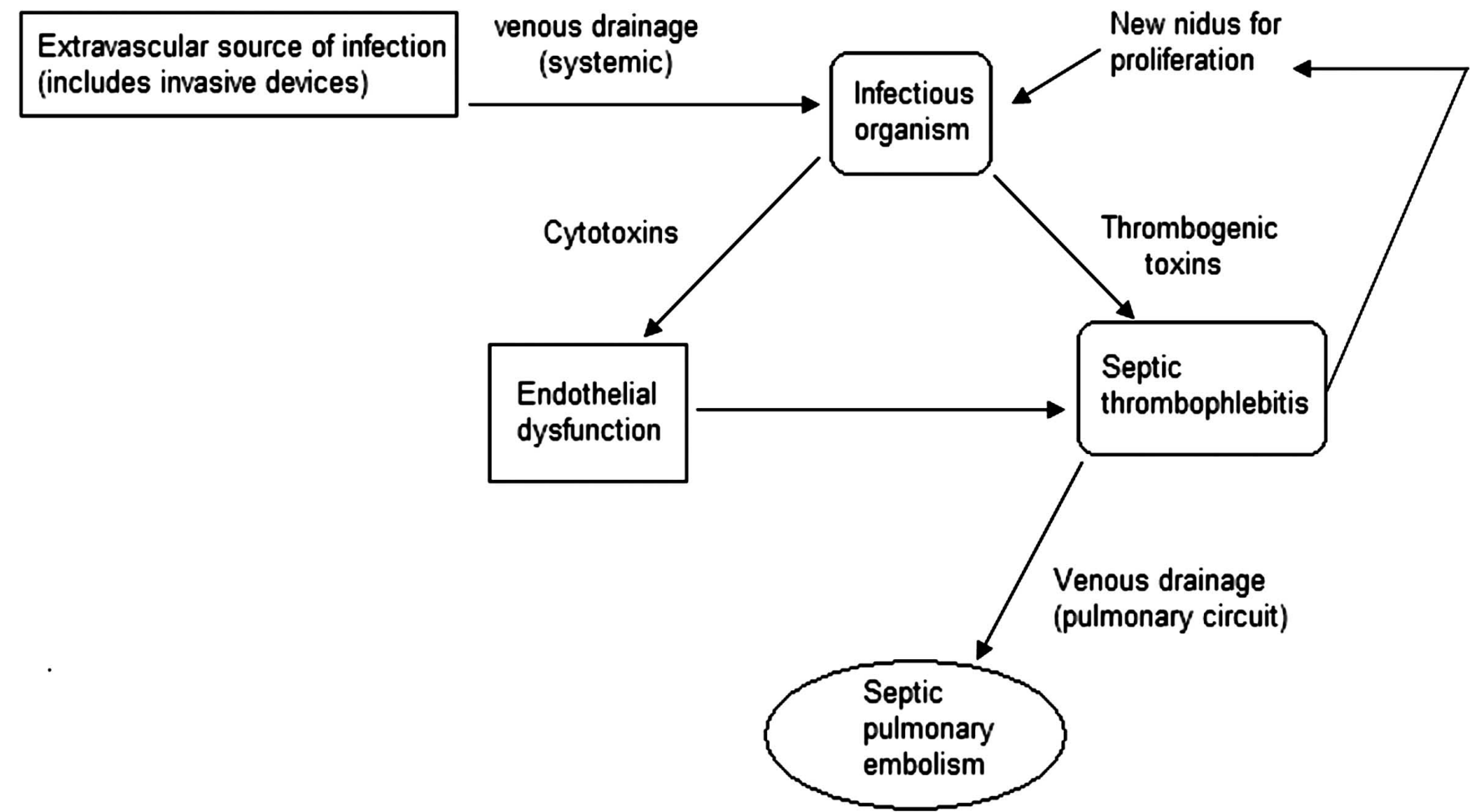

Fig. (2). Diagram that explains the possible pathophysiologic mechanisms of septic pulmonary embolism in the setting of septic thrombophlebitis 
devices (eg. intravenous catheters) and considering surgical intervention to remove purulent collections. Anticoagulation is the cornerstone of treatment for deep venous thrombosis, but when the patient presents with septic thrombophlebitis, its use becomes controversial. The purpose of this therapy is to diminish the severity and duration of symptoms during the acute thrombotic event by preventing thrombus propagation and extension, minimize the incidence of recurrent thrombosis and decrease the risk of pulmonary embolic complications. It is important to emphasize that it does not directly promote thrombus dissolution [16]. The main theoretical concern for the use of anticoagulation in septic thrombophlebitis would be the potential to increase the rates of septic embolism or to precipitate hemorrhagic conversion of metastatic lesions, particularly in the CNS. This seems to be extrapolated directly from the experience in infectious endocarditis [17]. However, to our knowledge, there is no literature to support this. None of our patients experienced these specific complications with the use of systemic anticoagulation along with proper antibiotic therapy. Ang and Brown [18] formally reviewed the available literature up to the decade of 1980 's, and found that there were no reports available to substantiate an increase in septic emboli in patients treated with anticoagulation in suppurative thrombophlebitis. A recent exhaustive systematic review [19] analyzed 14 case series, consisting of a total of 216 patients, to determine the role of intravenous heparin in combination with antibiotics for the treatment of septic thrombophlebitis.

The authors found that the use of heparin was associated with a low mortality and few reported serious adverse effects, which is concordant with our findings. Monitoring for hemorrhagic complications is always in order, for all patients receiving anticoagulants. One of our patients developed a hemopericardium; therefore anticoagulation was temporarily held. Given his poor respiratory reserve and risk for further embolism, decision was made to insert an inferior vena cava filter, which has been cited as a reasonable approach under these circumstances [20].

Several limitations should be considered in our study. Given its restrospective nature, selection bias was inevitable. Despite accurate microbiologic information, our data lacked molecular analysis of $S$. aureus strains for virulence factors. Although all patients underwent echocardiography to further interrogate for the possibility of endocarditis, not every patient underwent a transesophageal study, which is known to be more sensitive for the detection of such complication.

\section{CONCLUSIONS}

SPE continues to be a dreaded condition, with considerable morbidity and mortality, in the current antibiotic and advanced imaging era. Early diagnosis is key to improve patient outcomes.

The presence of septic thrombophlebitis in anatomical proximity to the main focus of infection has been recognized more frequently in cases of SPE. Infectious organisms with peculiar thrombogenic potential are also pivotal in the development of this complex. The role of anticoagulation in the setting of STP continues to be a matter of debate; recent studies have reported favorable outcomes with its use, and our experience is consistent with these findings. Comparative clinical trials evaluating these interventions are needed to make definitive recommendations for treatment of this syndrome.

\section{ACKNOWLEDGEMENT}

The authors wish to thank Ed Peterson for his assistance with the figures.

\section{CONFLICT OF INTEREST}

Declared none.

\section{REFERENCES}

[1] Fred HL, Harle TS. Septic pulmonary embolism. Chest 1969; 55: 483-6

[2] Gorenstein A, Gross E, Houri S, Gewirts G, Katz S. The pivotal role of deep vein thrombophlebitis in the development of acute disseminated staphylococcal disease in children. Pediatrics 2000; 106: E87.

[3] Volkow P, Cornejo-Juarez P, Arizpe-Bravo AB, Garcia-Mendez J, Baltazares-Lipp E, Perez-Padilla R. Catheter-related septic thrombophlebitis of the great central veins succesfully treated with low-dose streptokinase and antimicrobials. Thromb J 2005; 3: 11.

[4] Nourse C, Starr M, Munckhof W. Community acquired methicillin resistant $\mathrm{S}$. aureus causes severe disseminated infection and deep venous thrombosis in children: literature review and recommendations for management. J Pediatr Child Health 2007; 43: 656-61.

[5] LePage AA, Hers EP, Schears RM. Septic thrombophlebitis with acute osteomyelitis in adolescent children: a report of two cases and review of the literature. Int J Emerg Med 2008; 1: 155-9.

[6] Gonzalez BE, Teruya J, Mahoney DH, et al. Venous thrombosis associated with staphylococcal osteomyelitis in children. Pediatrics 2006; 117(5): 1673-9.

[7] Vander Have KL, Karmazyn B, Verma M, et al. Community acquired methicilin resistant $\mathrm{S}$. aureus in acute musculoskeletal infection in children: a game changer. J Pediatr Orthop 2009; 29(8): 927-31.

[8] Razonable RR, Rahman AE, Wilson WR. Lemierre's Syndrome variant: necrobacillosis associated with inferior vena cava thrombosis and pulmonary abscesses after trauma-induced leg abscess. Mayo Clin Proc 2003; 78: 1153-6.

[9] Holm K, Frick IM, Bjorck L, Rasmussen M. Activation of the contact system at the surface of Fusobacterium necrophorum represents a possible virulence mechanism in Lemierre' $\mathrm{s}$ syndrome. Infect Immun 2011; 79(8): 3284-90.

[10] Boyle-Vavra S, Daum RS. Community-acquired meticillinresistant S. aureus: the role of Panton-Valentine leucocidin. Lab Invest 2007; 87: 3-9.

[11] Kadhiravan T, Piramanayagan P, Banga A, Gupta R, Sharma SK. Lemierre's syndrome due to community-acquired meticillinresistant $\mathrm{S}$. aureus infection presenting with orbital cellulites: a case report. J Med Case Rep 2008; 2: 374.

[12] Boga C, Ozdogu H, Diri B, Oguzkurt L, Asma S, Yeral M. Lemierre's syndrome variant: S. aureus associated with thrombosis of both the right internal jugular vein and splenic vein after exploration of a river cave. J Thromb Thrombolysis 2007; 23: 1514.

[13] Shivashankar GH, Murukesh N, Varma MPS, Sharif IM, Glynn G. Infection by Panton-Valentine leukocidin-producing $\mathrm{S}$. aureus clinically mimicking Lemierre's syndrome. J Med Microbiol 2008; 57: 118-20.

[14] Jupiter JB, Ehrlich MG, Novelline RA, Leeds H, Keim D. The association of septic thrombophlebitis with subperiosteal abscesses in children. J Pediatr 1982; 101(5): 690-5

[15] Hota B, Lyles R, Rim J, et al. Predictors of clinical virulence in community onset methicillin-resistant S. aureus infections: the importance of USA 300 and pneumonia. Clin Infect Dis 2011; 53: 766-71.

[16] Nazir SA, Ganeshan A, Nazir S, Uberoi R. Endovascular treatment options in the management of lower limb deep venous thrombosis. Cardiovasc Intervent Radiol 2009; 32: 861-76. 
[17] Hart RG, Kagan-Hallet K, Joerns SE. Mechanisms of intracranial hemorrhage in infective endocarditis. Stroke 1987; 18: 1048-56.

[18] Ang AK, Brown OW. Septic deep vein thrombosis. J Vasc Surg 1986; 4: 563-6.

[19] Falagas ME, Vardakas KZ, Athanasiou S. Intravenous heparin in combination with antibiotics for the treatment of deep vein septic thrombophlebitis: a systematic review. Eur J Pharm 2007; 557: 938.

[20] Greenfield LJ, Proctor MC. Vena cava filter use in patients with sepsis: results in 175 patients. Arch Surg 2003; 138(11): 1245-8.

Received: March 14, 2012

Revised: April 15, 2012

Accepted: April 17, 2012

(C) Brenes et al.; Licensee Bentham Open.

This is an open access article licensed under the terms of the Creative Commons Attribution Non-Commercial License (http://creativecommons.org/licenses/by-nc/3.0/) which permits unrestricted, non-commercial use, distribution and reproduction in any medium, provided the work is properly cited. 